\title{
Considerations about Status of Severe Acquired Brain Injuries in Italy
} Renato Avesani, Francesca Dambruoso*, Rita Formisano, Antonio De Tanti, Salvatore Ferro, Nicola Smania, Laura Roncari and Elena Rossato

Department of Rehabilitation, Sacro Cuore Don Calabria Hospital, Negrar, Verona, Italy

Severe acquired brain injuries leads often to disability with consequences on families and social system as on sanitary system.

The creation of the "Italian National Registry of Severe Acquired Brain Injuries" [1], allowed the systematic collection of data about patients admitted to rehabilitation facilities in order to monitor the changes in demographic and clinical data during years and make some considerations about outcome and post discharge pathways.

The possibility to analyse data in a retrospective way highlights the increase, in the last years, of the mean age of the population affected by $\mathrm{ABI}$ with older people belonging to the non-traumatic group but also the awareness that subjects belonging to this group present greater disabilities both at admission and discharge from rehabilitation settings, compared to the traumatic subjects $[1,2]$.

There is an increase in survive after acquired brain injuries probably due to the amelioration of sanitary organization and more extensive rehabilitation networks.

However, according to the data collected, the number of patients in vegetative status (VS) at discharge from rehabilitation represent a significant rate, reaching the $37.85 \%$ in the group of patients affected by anoxic brain injuries.

With the aim to define outcome of specific subgroups of patients affected by ABI at discharge from rehabilitation wards, we considered the population, of the same sample of the previous study, that at time of admission in rehabilitation care was in vegetative status.

Recovery of consciousness and disability were evaluated using discharge Disability Rating Scale (DRS) score. At discharge, 53.11\% patients emerged from VS and most of them had a TBI; subjects with non-traumatic brain damage had the worst prognosis. Of those who emerged from VS $71.30 \%$ of TBI and $83.06 \%$ of NTBI presented an extremely severe disability.

Only $37.93 \%$ of subjects affected by TBI and $17.44 \%$ of patients affected by NTBI that presented extremely severe disability returned home after rehabilitation stay.

Despite almost a half of patients emerged from VS, a large amount of these subjects showed a great disability, and returning home is often not possible.

Furthermore, those patients often present a long survival, needing a life-long care, resulting in a familiar considerable financial commitment.

All those evidences lead us to the awareness that some demographic and clinical characteristics of patients affected by ABI are related with lower outcomes, defined as higher levels of disabilities.

The possibility to identify outcome predictors in advance to the admission in rehabilitation wards could be useful in order to plan the better pathway for the patient, a better allocation of resources directed on assistance or rehabilitation.

\section{References}

1. Avesani R, Roncari L, Khansefid M, Formisano R, Boldrini P, et al. (2013) The Italian National Registry of severe acquired brain injury: Epidemiological, clinical and functional data of 1469 patients. Eur J Phys Rehabil Med 49: 611-618.

2. Andriessen TM, Horn J, Franschman G, van der Naalt J, Haitsma I, et al. (2011) Epidemiology, severity classification and outcome of moderate and severe traumatic brain injury: A prospective multicenter study. J Neurotrauma 28: 20192031.
*Corresponding author: Francesca Dambruoso, Department of Rehabilitation, Sacro Cuore Don Calabria Hospital, Negrar, Verona, Italy, Tel: 393487340021; E-mail: francesca.dambruoso@sacrocuore.it

Received April 25, 2017; Accepted April 28, 2017; Published April 30, 2017

Citation: Avesani R, Dambruoso F, Formisano R, De Tanti A, Ferro S, et al. (2017) Considerations about Status of Severe Acquired Brain Injuries in Italy. Int J Neurorehabilitation 4: 257. doi: 10.4172/2376-0281.1000257

Copyright: $\odot 2017$ Avesani R, et al. This is an open-access article distributed unde the terms of the Creative Commons Attribution License, which permits unrestricted use, distribution, and reproduction in any medium, provided the original author and source are credited. 